\section{MS48-O2 Micro-XRPD, XRPD tomography and MA-XRPD: three related methods to better understand structural alterations in cultural heritage materials}

Koen H. Janssens ${ }^{1}$, Frederik Vanmeert ${ }^{1}$, Steven De Meyer ${ }^{1}$, Geert Van der Snickt ${ }^{1}$

\section{University of Antwerp, Department of Chemistry}

email: koen.janssens@uantwerpen.be

The (inner) structure of paintings may reveal changes made by the artists themselves or by others following them. Traditionally, this inner structure is examined by means of X-ray radiography and Infrared Reflectography. Both methods make use of deeply penetrative radiation; both give rise to black and white images that to some extent reflect the composition at or below the surface of the paintings. More powerful equivalents of these two methods are Macroscopic X-ray fluorescence imaging (MA-XRF) and hyperspectral imaging in the Visual and Near Infra-red (VNIR) region, allowing to collect a richer set of (spectroscopic) image data and construct false color images of paintings from which valuable information about the paint composition (at/below the surface) can be gleaned.

In many historical paintings, unwanted chemical transformations take place that may influence the general outlook/colors of part of a painting. Micro/nanoscopic spectrometric methods such as Raman/Infra-red microscopy and various forms of Electron Microscopy are useful to understand these transformations. Also the (combined) use of synchrotron radiation based methods such as microscopic X-ray fluorescence, X-ray absorption spectroscopy and X-ray diffraction is highly relevant to reconstruct the degradation mechanism of painters' pigments such as chrome yellow or red lead. We recently were able to unravel the transformation process that converts red lead (minium) in lead white (cerussite) via XRPD tomography by the identification of the intermediate crystalline phase, called plumbonacrite.

With the purpose of rendering the elemental mapping capability of MA-XRF more pigment-specific, based on a micro-focussed X-ray source equipped with focussing and monochromatizing mirrors, we have recently constructed a MA-XRPD scanning setup and have evaluated the possibility of using this for recording high-specific distributions of painters' pigments and some of their degradation products in a non-destructive manner.

By discussing a number of case studies involving $17^{\text {th }}$ and $19^{\text {th }}$ century paintings or paint samples from painters such as J. De Heem, E. Munch, H. Matisse and V. Van Gogh, the usefulness of highly-specific non-invasive analytical imaging at the macroscopic scale in combination with microscopic multimodal examination of minute paint fragments will be discussed and their relevance both for art historians and art conservators highlighted.

Keywords: Microscopic XRPD, Macroscopic XRPD imaging, synchrotron radiation, Van Gogh, XRPD tomography

\section{MS48-03 Crystallographic interpretation of mineral decompositions via Rietveld refinement strategy for clustering ancient ceramics}

Mohammadamin Emami ${ }^{1,2}$

1. Department of conservation and archaeology, Art University of Isfahan, Isfahan, Iran

2. Department of Building Material Chemistry, University Siegen, Germany

email: emami@chemie.uni-siegen.de

Ceramics in the antiquity have a very complex structure according to their mineralogical constituents and their accidental or experimental manufacturing processes. The sintered body of such objects suggest a well-organized technological now-how at this time. Ceramics are always classified by archaeologists according to their external features and therefor the investigations are done mostly comparatively.

This study focuses on the exact quantitative phase composition of a collection of ceramics from 1250 millennium BC. Mineralogical properties of the crystalline phases are studied by means of polarization microscopy and X-ray diffraction inclusive Rietveld refinement strategy in order to get information about the different crystalline phase constituents via different firing conditions due to the - thermodynamically mineralogical stability exchange.

According to the crystalline phase decompositions which have been carried out in the ceramic texture, the technology has been changed by means of different firing condition. Clustering the data proved that the manufacturing process mostly changed due to the firing condition and not only by means of raw material usage. Due to the results, ceramics can be classified in archetypal groups.

Keywords: ceramics, Rietveld refinement 